\title{
NATURALISTIC DRIVING: USER AND TASK ANALYSIS
}

\author{
P.Ward ${ }^{1}$, P.A. Hancock ${ }^{1,2}$, H.C.N. Ganey ${ }^{1}$, and J.L. Szalma ${ }^{1,2}$ \\ ${ }^{1}$ Department of Psychology, University of Central Florida \\ ${ }^{2}$ Institute for Simulation and Training \\ Department of Psychology \\ University of Central Florida \\ P.O. Box 161390, Orlando, FL 32816-1390 \\ E-mail: hganey@mail.ucf.edu
}

\begin{abstract}
Summary: Cognitive Task Analysis and methods for analyzing Naturalistic Decision Making are powerful tools that can be applied to transportation research. In conjunction with simulators, these methods allow increased understanding of real user interactions with their in-vehicle systems, and the decision processes involved in the operational aspects of driving, navigating, and using infotainment support systems. Adopting this approach facilitates investigation of driver performance under a range of workload and stress conditions, which supports future development of a prototypical model that will encapsulate the cognitive and perceptual-motor demands of driving in the presence of situational stressors under both high- and low-workload conditions.
\end{abstract}

\section{INTRODUCTION}

Driving is a common task for most people who meet the eligibility requirements for licensure. The ability to drive and the possession of a vehicle are symbols of freedom, allowing drivers to come and go as they please. However, this freedom is accompanied by the risk of collision and injury. Thus, driving is an activity that is potentially lethal to drivers, passengers, and pedestrians alike, although most drivers hold expectations that such events will not occur. Further, most drivers receive minimal training, and the training they do experience typically occurs under conditions that only marginally simulate the multitude of 'on-road' and 'in-vehicle' demands. Even for those who receive extensive training (e.g., professional drivers), experiences of cognitive overload from the simultaneous use of operational, navigational, and in-vehicle infotainment support systems, as well as related ancillary tasks such as the use of cellular telephones, can negatively affect performance and significantly increase driver risk.

To date, much of the extant 'laboratory' research has examined limited aspects of the perceptual or motor demands faced by drivers. This research typically isolates these variables from other integral components, limiting the ability of such studies to explain real-world driver performance (see Klein, Vincent \& Isaacson, 2001). This is a vital issue, since there are a variety of performance measures for the driving task (Jerome et al., 2001). In this paper we suggest a practical approach to measuring driver performance using techniques drawn from cognitive task analysis and naturalistic decision-making research. 


\section{A POSSIBLE COURSE OF ACTION}

'Naturalistic Decision Making' (NDM) was developed as an approach to studying human performance with the specific intention of addressing how individual's use their experience to facilitate performance in actual 'field settings' (e.g., see Zsambok \& Klein, 1997). This approach is typically utilized for studying task performance in 'real world' environments rather than for laboratory decision-making tasks. In addition, factors such as time pressure, spatial and temporal uncertainty, and dynamic environmental change, interact to increase the complexity of delineating the nuances of 'on the road' driver performance (see Klein, 1999). Though the NDM approach has its challenges (see Cannon-Bowers et al., 1996), we believe these weaknesses can be addressed with a thorough task analysis of the interaction between user and in-vehicle systems and of the underlying processes involved in driving.

Cognitive task analysis (CTA) has been proposed as a method which determines the cognitive processes and skills required for a high level of task performance where tasks are cognitively complex and environments are dynamic, uncertain, and similarly complex (see O'Hare, Wiggins, Williams, \& Wong, 1998). Several variations of the method exist (see Seamster, Redding, \& Kaempf, 1994), but a well-known CTA technique used to study naturalistic decision-making is the Critical Decision Method (Klein, Calderwood \& Macgregor, 1989). The essence of this retrospective interview technique lies in its effective use of cognitive probes to elicit knowledge and strategies used in expert decision-making.

Simulation is a powerful tool for examining performance in naturalistic settings. That is, ecologically valid, high-fidelity and functional driving environments can be created to replicate 'on-road' conditions which afford the opportunity for representative behavior to be studied under controlled and replicable conditions, allowing a range of quantitative and qualitative data to be collected (Klein, 1999).

Recent NDM research in the domain of driving using CTA interview methods examined the proficiency of young and long-term drivers to determine the types of decisions and nature of challenge encountered (Klein, Vincent, \& Isaacson, 2001). Differences in decision-making were observed between groups, with more experienced drivers being more attentive to environmental cues and novice drivers using heuristics and more basic signals. The study by Klein et al. (2001) underscores the importance of evaluating performance under context-specific real world or simulated conditions that impose relevant cognitive and stress-related demands. Such evaluation is essential if realistic behaviors are to be observed in driving research.

\section{REFERENCES}

Cannon-Bowers, J.A., Salas, E., \& Pruitt, J.S. (1996). Establishing the boundaries of a paradigm for decision-making research. Human Factors, 38(2), 193-205.

Jerome, C.J., Ganey, H.C.N., Mouloua, M., \& Hancock, P.A. (2001). Driver Workload Response to In-Vehicle Device Operations. International Journal of Occupational Safety and Ergonomics, 8(4), 539-548.

Klein, G. (1999). Applied decision making. Found in: P.A. Hancock (Ed.) Human performance and ergonomics. (pp. 87-107). San Diego: Academic Press, Inc. 
Klein, G. Calderwood, R. \& Macgregor, D. (1989). Critical decision method for eliciting knowledge. IEEE Transactions on Systems, Man, \& Cybernetics, 19(3), 462-472.

Klein, H.A., Vincent, E.J., \& Isaacson, J.J. (2001). Driving proficiency: The development of decision skills. Found in: E. Salas \& G. Klein, (Eds.) Linking expertise and naturalistic decision making. (pp. 303-320), Lawrence Erlbaum Associates, Mahwah, NJ.

O’Hare, D., Wiggins, M., Williams, A., \& Wong W. (1998). Cognitive task analyses for decision centred design and training. Ergonomics, 41(11), 1698-1718.

Seamster, T.L., Redding, R.E., Kaempf, G.L. (2000). A skill-based cognitive task analysis framework. Found in: J.M. Schraagen, S.F. Chipman \& V.L. Shalin (Eds) Cognitive task analysis, pp. 135-146. Mahwah, NJ: Lawrence Erlbaum Associates, Inc.

Zsambok, C.E., \& Klein, G. (1997). Naturalistic decision making. Expertise: Research and applications. Hillsdale, NJ: Lawrence Erlbaum Associates, Inc. 